\title{
Vibration modeling of structural fuzzy with continuous boundary
}

\author{
Friis, Lars; Ohlrich, Mogens
}

Published in:

Journal of the Acoustical Society of America

Link to article, DOI:

$10.1121 / 1.2823498$

Publication date:

2008

Document Version

Publisher's PDF, also known as Version of record

Link back to DTU Orbit

Citation (APA):

Friis, L., \& Ohlrich, M. (2008). Vibration modeling of structural fuzzy with continuous boundary. Journal of the Acoustical Society of America, 123(2), 718-728. https://doi.org/10.1121/1.2823498

\section{General rights}

Copyright and moral rights for the publications made accessible in the public portal are retained by the authors and/or other copyright owners and it is a condition of accessing publications that users recognise and abide by the legal requirements associated with these rights.

- Users may download and print one copy of any publication from the public portal for the purpose of private study or research.

- You may not further distribute the material or use it for any profit-making activity or commercial gain

- You may freely distribute the URL identifying the publication in the public portal

If you believe that this document breaches copyright please contact us providing details, and we will remove access to the work immediately and investigate your claim. 


\title{
Vibration modeling of structural fuzzy with continuous boundary
}

\author{
Lars Friis ${ }^{\mathrm{a})}$ \\ Acoustic Technology, Ørsted DTU, Technical University of Denmark, Building 352, \\ DK-2800 Kgs. Lyngby, and Widex A/S, Ny Vestergaardsvej 25, DK-3500 Vaerloese, Denmark \\ Mogens Ohlrich ${ }^{\mathrm{b})}$ \\ Acoustic Technology, Ørsted DTU, Technical University of Denmark, Building 352, \\ DK-2800 Kgs. Lyngby, Denmark
}

(Received 29 June 2007; revised 5 November 2007; accepted 20 November 2007)

\begin{abstract}
From experiments it is well known that the vibration response of a main structure with many attached substructures often shows more damping than structural losses in the components can account for. In practice, these substructures, which are not attached in an entirely rigid manner, behave like a multitude of different sprung masses each strongly resisting any motion of the main structure (master) at their base antiresonance. The "theory of structural fuzzy" is intended for modeling such high damping. In the present article the theory of fuzzy structures is briefly outlined and a method of modeling fuzzy substructures examined. This is done by new derivations and physical interpretations are provided. Further, the method is extended and simplified by introducing a simple deterministic approach to determine the boundary impedance of the structural fuzzy. By using this new approach, the damping effect of the fuzzy with spatial memory is demonstrated by numerical simulations of a main beam structure with fuzzy attachments. It is shown that the introduction of spatial memory reduces the damping effect of the fuzzy and in certain cases the damping effect may even be eliminated completely. (0) 2008 Acoustical Society of America.
\end{abstract}

[DOI: $10.1121 / 1.2823498$ ]

PACS number(s): 43.40.At, 43.40.Tm [DF]

Pages: $718-728$

\section{INTRODUCTION}

It is commonly known from experiments that vibrations of a complicated system consisting of a main structure and a large number of small attached resonant substructures often appears to be more damped than the main structure's damping properties would imply. Already in 1928 Ormondroyd and Den Hartog ${ }^{1}$ revealed that a "dynamic absorber" could produce a considerable reduction in vibration level, however, only in a relatively narrow frequency band around its natural frequency. To be effective such an auxiliary system is usually attached at the forcing point of the main structure. And the auxiliary system is predominantly a reflecting device controlled by its stiffness and mass, although its bandwidth of influence can be slightly increased by a tuning, which involves small adjustments of both stiffness and damping of the device. Therefore, the term "dynamic neutralizer" has been adopted. ${ }^{2}$ For a more recent and detailed treatment of this subject the reader is referred to the monograph by Mead. ${ }^{3}$ It took almost 60 years before Soize ${ }^{4}$ and Chabas et $a .^{5}$ suggested that attached resonant substructures, in effect, behave like a multitude of dynamic neutralizers with different natural frequencies that introduce a high damping in the main structure over a broader frequency range.

Many complicated engineering systems consist basically of an outer shell- or a box-like master structure and a com-

\footnotetext{
${ }^{a)}$ Electronic mail: 1f@oersted.dtu.dk

${ }^{b)}$ Electronic mail: mo@oersted.dtu.dk
}

plicated internal structure. Examples of such structures varying from small to large sizes are electromechanical hearing aids, machines, aircraft, and ship hulls. The outer master structure is often well defined and its vibration can be predicted using conventional methods of vibrational analysis. In contrast, the dynamic properties of the internals may only be partly known and therefore their dynamics and influence have to be modeled by using an alternative method such as that offered by "fuzzy structure theory." ${ }^{,-9}$ This theory is intended for an overall and simple prediction of the vibration of the master structure, and the theory considers the internal parts as a single or several independent "fuzzy substructures," which are known in some statistical sense only.

In some systems the fuzzy substructures are attached to the master through a continuous boundary or junction. This could, for example, be line-coupled machinery in a ship hull or passenger seats and luggage compartments attached to the main structure of an airplane. The continuous connection boundary implies that spatial coupling within the fuzzy has to be considered, and it is only in special cases that this coupling can be neglected.

Often the motion of the continuous junctions is varying significantly with position due to the spatial variation of vibration in the master structure, and spatial coupling forces in the fuzzy have to be accounted for. The present article addresses this problem of including spatial coupling in the modeling of structural fuzzy.

With frequency or vibration wavelength as a parameter, Fig. 1 shows three scenarios, each with three different cases 

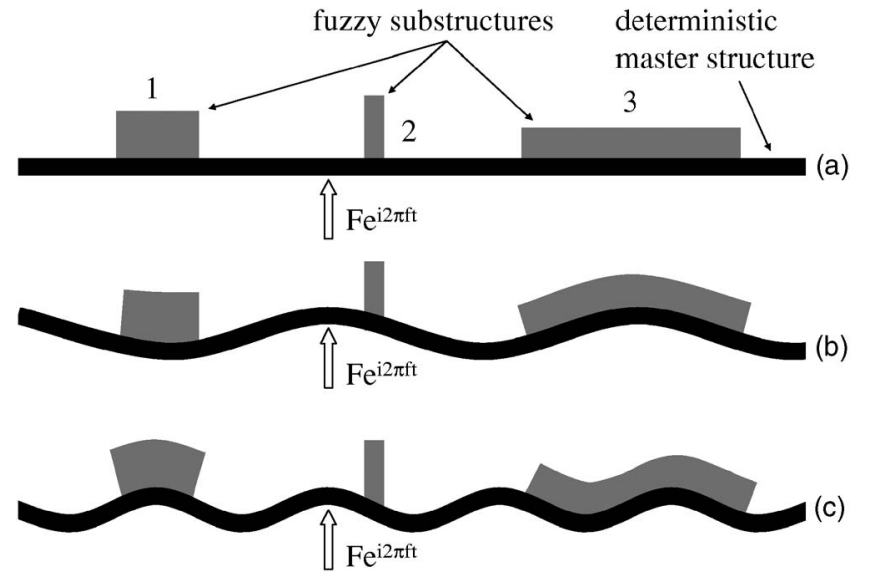

FIG. 1. Three different fuzzy substructures attached to a master structure undergoing harmonic vibration: (a) low frequency rigid body motion, (b) low frequency wave motion, and (c) mid-frequency wave motion.

of fuzzy substructures attached to a master. It is assumed that a time harmonic force of amplitude $F$ and angular frequency $\omega=2 \pi f$ excites the master and generates vibration in the whole system. At very low frequencies the master structure vibrates as a rigid body in translational motion [see Fig. 1(a)], and the junction displacement at the boundary of the fuzzy substructures is almost constant. This implies that the spatial coupling within each substructure has no significant effect on the response of the system as a whole. Now, increasing the excitation frequency introduces elastic motion in the master structure and hence at the interface with the fuzzy. When the vibration wavelength of the master becomes comparable with the dimensions of the fuzzy connection area then the spatial coupling begins to take effect. This is the case in Fig. 1(b) where the boundary displacement of substructure 3 is varying whereas those of substructures 1 and 2 are nearly constant. In Fig. 1(c) the frequency has been increased further and the boundary displacements of both substructures 1 and 3 are varying, whereas the boundary displacement of substructure 2 remains close to constant.

Fuzzy structure theory was originally developed by Soize and co-worker and presented in a series of papers ${ }^{4-7}$ during a 10-year period starting in 1986. These papers involve probabilistic concepts in order to take the model uncertainties into account. In attempts to explain the main ideas behind the theory, these papers have been subject to numerous simplifications, interpretations, and extensions during the last 20 years.

In particular, Pierce et al. ${ }^{8}$ and Strasberg and Feit ${ }^{9}$ have introduced more simple and deterministic methods in order to predict the average responses of the master. In these methods the main parameter describing the fuzzy is taken to be the distribution of resonating mass per unit frequency. One of the crucial steps in applying fuzzy structure theory is the very estimation of this mass distribution. Both Soize ${ }^{10,11}$ and Pierce $^{12}$ have addressed this problem throughout the last 10 years.

In 1993 Soize briefly presented a method ${ }^{6}$ for including spatial memory in the modeling of structural fuzzy with continuous boundaries. Despite this, elaborating literature has so far mainly been concerned with structural fuzzy without spa- tial coupling effects. However, in order to utilize Soize's innovative theory on structural fuzzy with spatial memory, there is a strong need for a detailed examination of the suggested method in addition to a presentation of necessary supplementary derivations. Moreover, a simple method for implementing the structural fuzzy is still absent in the open literature. The objective of the present article is to extend Soize's theory by using a simplifying approach, which to some extent is based on the methods introduced by Pierce. et al., ${ }^{8}$ and Strasberg and Feit. ${ }^{9}$ The outline of the present article is as follows. Succeeding a brief outline of the theory of fuzzy structures in Sec. II, the method of including spatial memory is discussed in details and extended in Sec. III; this includes (i) derivation of the boundary impedance of Soize's spatial oscillator, (ii) derivation of the boundary impedance of an infinite number of identical spatial oscillators evenly distributed on the fuzzy connection area, and (iii) introduction to Soize's local equivalent oscillator and equivalent coupling factor and a presentation of new physical interpretations. Further, in Sec. IV we present a new approach for determining the boundary impedance of structural fuzzy with spatial memory. Finally, numerical simulations based on this approach are presented in Sec. V in order to illustrate the damping effects of structural fuzzy, which includes spatial memory.

\section{STRUCTURAL FUZZY WITHOUT SPATIAL MEMORY}

The purpose of the fuzzy structure theory is to model the overall vibrational response of a master structure, which has an attachment of one or more resonant substructures. A fuzzy substructure is considered as being composed of many simple oscillators resonating at different frequencies and being attached to the master at their base. When modeling such a system it is an advantage to separate the fuzzy from its master. Each fuzzy substructure is conveniently represented in terms of its boundary impedance. ${ }^{4,5}$ Using this approach the modeling of the response of the master with fuzzy attachments can be achieved without exceeding the number of degrees of freedom required for predicting the response of the master structure itself. As mentioned previously, one can neglect the spatial coupling effects in the fuzzy when a fuzzy substructure of multiple resonators is connected to the master over a small length or over a small area of virtually constant motion.

This is illustrated in Fig. 2 showing a fuzzy substructure, which is modeled by $N$ simple oscillators that is attached locally at an area $A$ of the master structure. An expression for the total boundary impedance $\underline{z}_{\text {fuzzy }}(f)$ of this substructure can be derived by superposition and by assuming, say, that the $n$th simple oscillator of the fuzzy is defined by the mass $M_{n}$, the undamped resonance frequency $f_{r, n}$ and the loss factor $\eta$. By introducing the complex stiffness of the oscillator $\underline{s}_{n}=s_{n}(1+i \eta)$, where $s_{n}=\left(2 \pi f_{r, n}\right)^{2} M_{n}$ is the impedance of the oscillator $\underline{Z}_{n}=\underline{F}_{n} / \underline{v}_{n}$ at the attachment base yields ${ }^{9}$ 


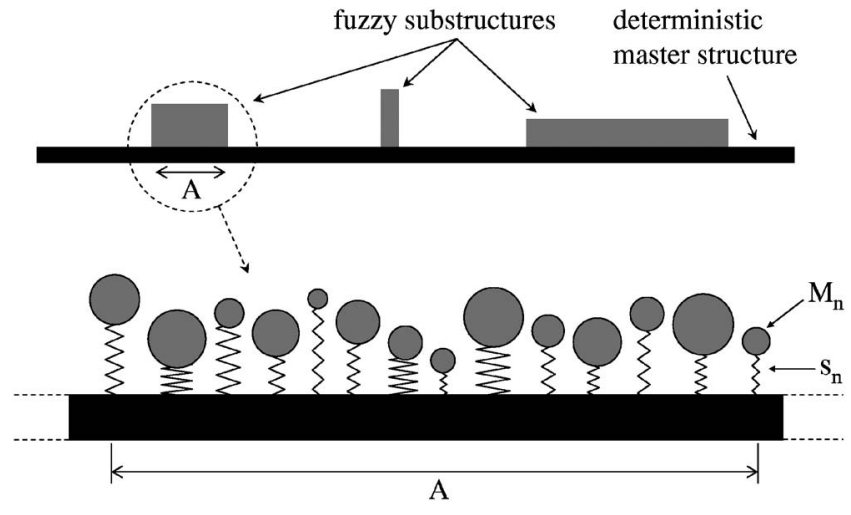

FIG. 2. Master structure exemplifying an attached fuzzy substructure, which is composed of $N$ simple oscillators resonating at different frequencies and without spatial memory.

$$
\begin{aligned}
\underline{Z}_{n}= & \frac{\underline{s}_{n}}{i \omega}\left(1-\frac{\underline{s}_{n}}{\underline{s}_{n}-\omega^{2} M_{n}}\right)=-i 2 \pi f\left(\frac{f_{r, n}^{2}}{f^{2}}\right)(1+i \eta) \\
& \times M_{n}\left(1-\frac{f_{r, n}^{2}(1+i \eta)}{f_{r, n}^{2}(1+i \eta)-f^{2}}\right) .
\end{aligned}
$$

Figure 3 shows the frequency variation of this oscillator impedance in a normalized form for different values of spring damping $\eta$. Below and above its resonance frequency the oscillator is, respectively, mass controlled and spring controlled. Further, at resonance of the oscillator, where the impedance is very large and almost purely real, it will strongly oppose any movements of its base. It is this particular feature of the oscillator, which results in the damping effect of the fuzzy substructure.

Generally, the different oscillators of a fuzzy substructure have different masses and natural frequencies and they are attached randomly to the master structure within the considered fuzzy connection area. Also, the total mass of all the oscillators equals the mass of the fuzzy substructure $M_{\text {fuzzy }}$
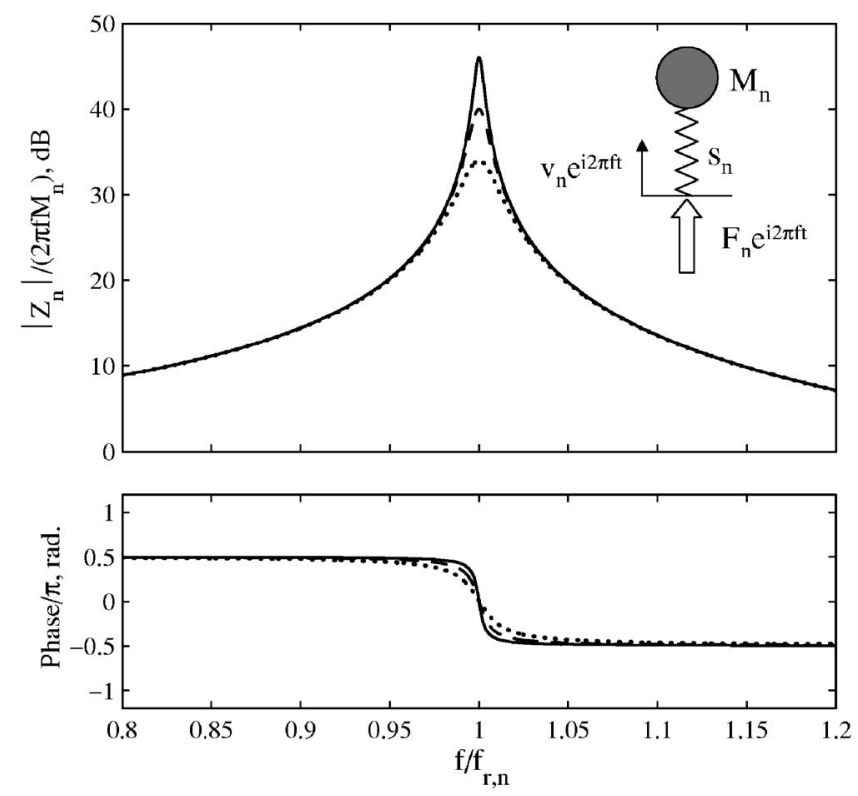

FIG. 3. Frequency variation of normalized impedance, $\underline{Z}_{n} /\left(2 \pi f M_{n}\right)$ for different values of spring damping $\eta$ :,$- 0.005 ;---, 0.01 ; \cdots, 0.02$.
$=\sum_{n=1}^{N} M_{n}$. Below a certain frequency, say $f_{r, \text { lower }}$, the oscillators will all be mass controlled. By increasing the frequency gradually from $f_{r \text {,lower }}$ to an upper limit, say $f_{r \text {,upper }}$, the oscillators will resonate one by one. Now, at each frequency within this "resonant" frequency band $f_{r \text {,lower }} \leqslant f_{r} \leqslant f_{r \text {,upper }}$ at least one oscillator will be close to its base antiresonance and it will therefore oppose the motion of the master. If the oscillators are attached close to one another within the area $A$, which has a nearly constant displacement, then the effective boundary impedance of all the oscillators, $\underline{z}_{\text {fuzzy }}(f)$, can be approximated by the sum of each oscillator's impedance $\underline{Z}_{n}(f)$ divided by the attachment area $A$ :

$$
\begin{aligned}
\underline{Z}_{\text {fuzzy }}(f)= & \frac{1}{A} \sum_{n=1}^{N} \underline{Z}_{n}(f)=-\frac{i 2 \pi f}{A} \sum_{n=1}^{N}\left(\frac{f_{r, n}^{2}}{f^{2}}\right)(1+i \eta) \\
& \times M_{n}\left(1-\frac{f_{r, n}^{2}(1+i \eta)}{f_{r, n}^{2}(1+i \eta)-f^{2}}\right) .
\end{aligned}
$$

This boundary impedance, however, requires specific knowledge about the properties of each oscillator and it is therefore conveniently replaced by an asymptotic and smoothed version. ${ }^{8,9}$ This is obtained by considering infinitely many oscillators resonating within the frequency band of $f_{r \text {,lower }} \leqslant f_{r} \leqslant f_{r \text {,upper }}$ and having a total mass $M_{\text {fuzzy }}$. This smoothed impedance yields ${ }^{8,9}$

$$
\begin{aligned}
\underline{z}_{\text {fuzzy }}(f)= & -\frac{i 2 \pi f}{A} \int_{f_{r, \text { lower }}}^{f_{r \text {,upper }}}\left(\frac{f_{r}^{2}}{f^{2}}\right)(1+i \eta) \\
& \times m_{\text {fuzzy }}\left(f_{r}\right)\left(1-\frac{f_{r}^{2}(1+i \eta)}{f_{r}^{2}(1+i \eta)-f^{2}}\right) d f_{r},
\end{aligned}
$$

where the quantity $m_{\text {fuzzy }}\left(f_{r}\right) d f_{r}$ represents the mass resonating between the frequencies $f_{r}$ and $f_{r}+d f_{r}$; this means that the total mass of the fuzzy substructure now is expressed as

$$
M_{\text {fuzzy }}=\int_{f_{r \text {, lower }}}^{f_{r, \text { upper }}} m_{\text {fuzzy }}\left(f_{r}\right) d f_{r} .
$$

The damping effect of the fuzzy substructure is mainly governed by this frequency dependent resonating mass distribution $m_{\text {fuzzy }}\left(f_{r}\right)$., , Methods for finding this parameter were suggested by Soize ${ }^{10,11}$ and Pierce, ${ }^{12}$ and different prototype mass distributions were proposed by Pierce $e t$ al. ${ }^{8}$ and Strasberg and Feit. ${ }^{9}$

As an example of the damping effect of structural fuzzy Fig. 4 shows computed results for the velocity vibration response per unit harmonic force, $\underline{Y}=\underline{v} / \underline{F}$, of a flexurally vibrating master beam, free in space, both without and with an attached substructure represented by 16 different simple oscillators. The resonance frequencies of these oscillators are spaced in geometric progression from 500 to $5000 \mathrm{~Hz}$. Further, the oscillators have identical point masses, weighting in total $10 \%$ of the master beam and a spring loss factor of $\eta$ $=0.05$. It is clearly observed that the attached substructure has a strong effect on the master response; this is seen to be reduced considerable over a broad band of frequencies and by up to $18 \mathrm{~dB}$ around $1300 \mathrm{~Hz}$. Further, it is seen that this substructure can be modeled successfully as a smoothed structural fuzzy by using the expression in Eq. (3). An ap- 

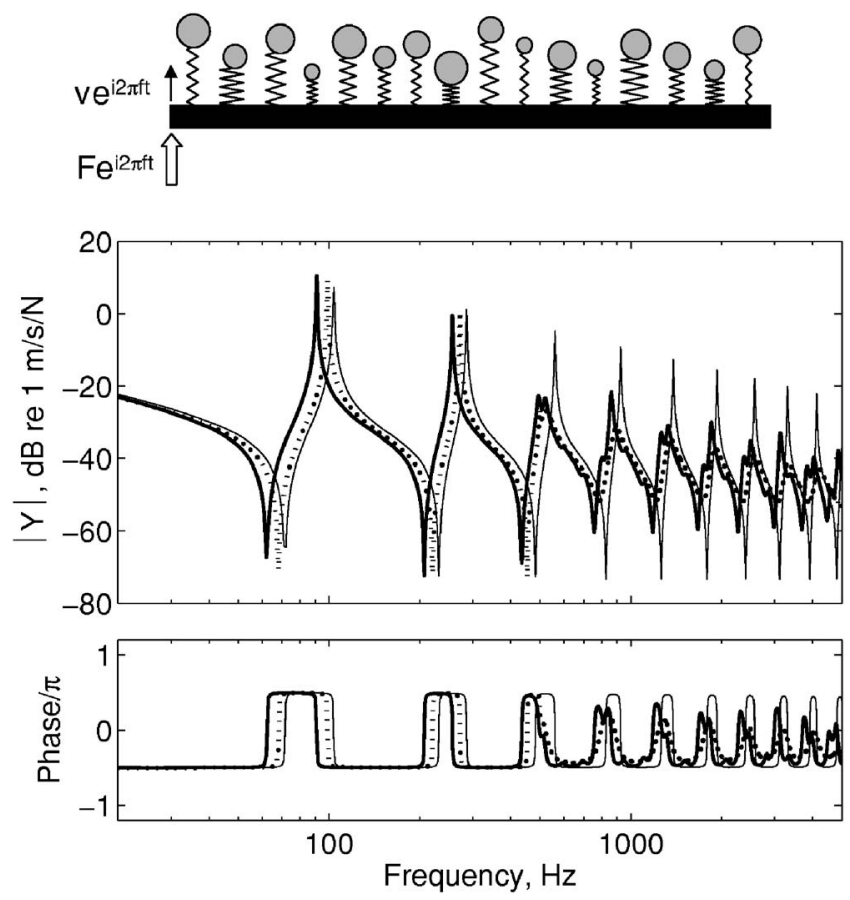

FIG. 4. Vibration velocity response per unit harmonic force, $\underline{Y}=\underline{v} / \underline{F}$, of a master beam free in space. Beam: - , without structural fuzzy; $\cdots$, with an attached fuzzy substructure represented by 16 simple oscillators, -; with a smoothed layer of structural fuzzy without spatial memory.

proximate condition for using this expression is suggested in Ref. 9 as $\eta>2 \Delta f_{r, n} / f_{r, n}$ where $\Delta f_{r, n}$ is the spacing between adjacent resonance frequencies. In other words this very strict condition requires that $\Delta f_{r, n}<\Delta f_{3 \mathrm{~dB}} / 2$ where $\Delta f_{3 \mathrm{~dB}}$ denotes the $3 \mathrm{~dB}$ bandwidth of the oscillator at resonance. However, in the present example it applies that the spacing between resonances is $\Delta f_{r, n} \approx 65 \cdot \Delta f_{r, n, 3 \mathrm{~dB}} / 2$ around $2000 \mathrm{~Hz}$, that is, 65 wider than the suggested requirement. It is therefore evident that acceptable results can be obtained with a much relaxed condition.

\section{SOIZE'S STRUCTURAL FUZZY WITH SPATIAL MEMORY}

\section{A. Soize's spatial oscillator}

Consider a fuzzy substructure connected to the master through a continuous boundary. A fuzzy substructure is generally attached to the master within an area, but for the sake of simplicity we shall here consider a fuzzy attached to the master through a one-dimensional boundary of length $L_{\text {fuzzy }}$. Soize incorporates a spatial memory in the structural fuzzy by introducing a "spatial oscillator" as sketched in Fig. 5(a). A structural fuzzy with spatial memory is composed of $N$ different sets $(n \in[1, N])$ of such spatial oscillators. Each of these $N$ sets consists of infinitely many identical spatial oscillators spread on the fuzzy connection area-or length. Let us first consider only one spatial oscillator, say the $i$ th $(i$ $\in[1, \infty])$ of the $n$th set of spatial oscillators of a fuzzy substructure, see Fig. 5(a). This oscillator is defined by the resonance frequency $f_{r, n}$, the lossfactor $\eta$ and the point mass $M_{n, i}$ located at position $x^{\prime}$. Further, the point mass is assumed supported by spring elements of stiffness density $\underline{s}_{\varepsilon, n, i}\left(x^{\prime}, x\right)$

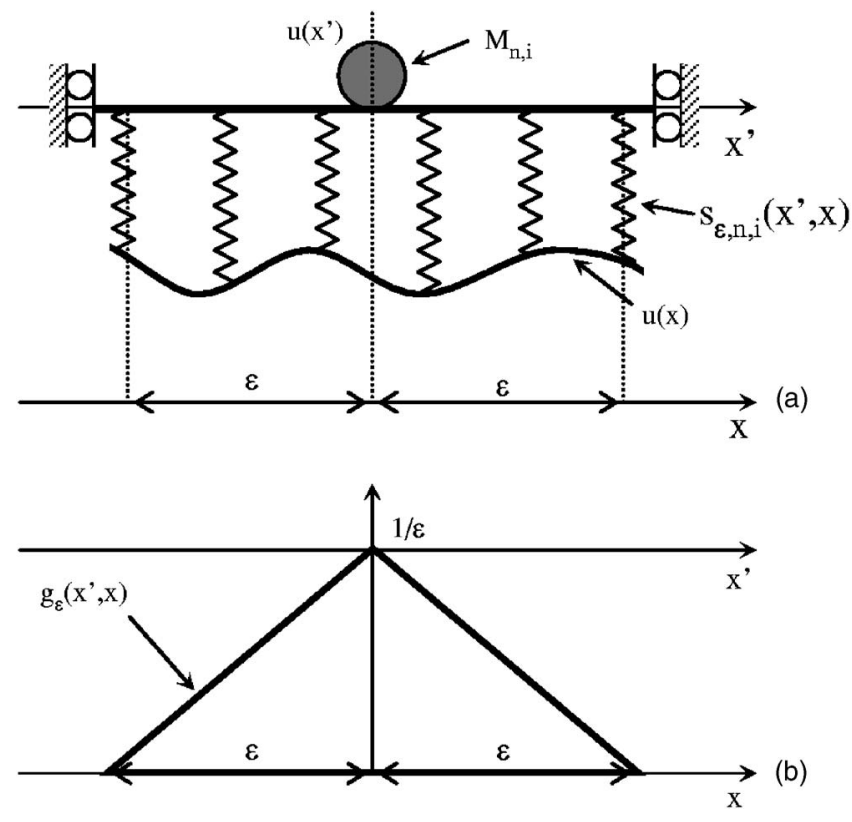

FIG. 5. Fuzzy oscillator with spatial coupling. (a) Oscillator attached to a boundary of motion $\underline{u}(x)$ and (b) stiffness density distribution $g_{\varepsilon}\left(x^{\prime}, x\right)$ of the oscillator spring elements.

that are attached to the master structure at different positions $x \in\left[x^{\prime}-\varepsilon, x^{\prime}+\varepsilon\right]$. Moreover, the spatial variation of the vibration displacement of the master is shown as $u(x)$ in Fig. 5(a). The actual width $2 \varepsilon$ of the distributed springs Soize denotes "the spatial memory" and the stiffness density he defines as ${ }^{6}$

$$
\underline{s}_{\varepsilon, n, i}\left(x^{\prime}, x\right)=\underline{s}_{n, i} g_{\varepsilon}\left(x^{\prime}, x\right)=\left(M_{n, i} \omega_{r, n}^{2}\right)(1+i \eta) g_{\varepsilon}\left(x^{\prime}, x\right) .
$$

Here, $\underline{s}_{n, i}$ is the total complex stiffness of the $i$ th oscillator belonging to the $n$th set, and the quantity $g_{\varepsilon}\left(x^{\prime}, x\right)$ is an even and positive-valued function of area 1 . As a onedimensional spatial memory Soize suggests a simple triangular distribution function $g_{\varepsilon}\left(x^{\prime}, x\right)$ as shown in Fig. 5(b). This is determined as

$$
g_{\varepsilon}\left(x^{\prime}, x\right)=\frac{\varepsilon-\left|x^{\prime}-x\right|}{\varepsilon^{2}} 1_{\left[x^{\prime}-\varepsilon, x^{\prime}+\varepsilon\right]}
$$

where $1_{\left[x^{\prime}-\varepsilon, x^{\prime}+\varepsilon\right]}$ is a function, which is equal to 1 for $x$ $\in\left[x^{\prime}-\varepsilon, x^{\prime}+\varepsilon\right]$ and which is 0 elsewhere. As the area under the curve $g_{\varepsilon}\left(x^{\prime}, x\right)$ is 1 , the oscillator in Fig. 5(a) has the same natural frequency $f_{r, n}$ as the simple oscillator with mass $M_{n}$ and stiffness $s_{n}$ that was considered in Sec. II. From the expression in Eq. (6) it is seen that the distribution $g_{\varepsilon}\left(x^{\prime}, x\right)$ only is dependent on the difference $x^{\prime}-x$ and therefore it can be written as

$$
g_{\varepsilon}\left(x^{\prime}-x\right)=\frac{\varepsilon-\left|x^{\prime}-x\right|}{\varepsilon^{2}} 1_{\left[x^{\prime}-\varepsilon, x^{\prime}+\varepsilon\right]}
$$

where it applies that $g_{\varepsilon}\left(x^{\prime}-x\right)=g_{\varepsilon}\left(x-x^{\prime}\right)$ and further that $\underline{s}_{\varepsilon, n, i}\left(x^{\prime}-x\right)=\underline{s}_{\varepsilon, n, i}\left(x-x^{\prime}\right)$.

\section{B. The $n$th set of spatial oscillators}

Next, consider the infinitely many identical oscillators belonging to the $n$th set. Let us assume that the oscillators 


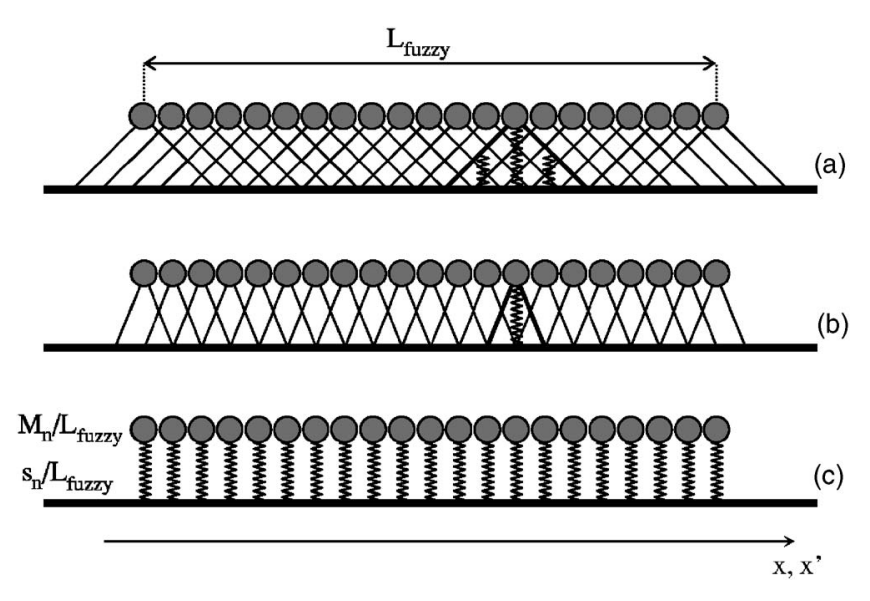

FIG. 6. Structural fuzzy attached to the master; fuzzy with: (a) high spatial memory, (b) small spatial memory, and (c) no spatial memory.

are distributed evenly over the fuzzy boundary, so that each location $x^{\prime}$ is associated with a point mass $M_{n, i}$. This is illustrated in Fig. 6 where each one of the oscillators representing the one sketched in Fig. 5(a) is depicted as a point mass and the associated triangular stiffness distribution. The point masses can vibrate independently whereas the spring elements overlap spatially at the connection boundary.

These infinitely many identical oscillators constitute the $n$th contribution to the total boundary impedance of the homogeneous fuzzy substructure. The total mass and total stiffness of all the oscillators of the $n$th set are given as $M_{n}$ and $\underline{s}_{n}$, respectively, so that $M_{n, i}=M_{n} / L_{\text {fuzzy }}$ and $\underline{s}_{n, i}=\underline{s}_{n} / L_{\text {fuzzy }}$. Figure 6(a) illustrates the case of spatial oscillators with a large width — or a high spatial memory-beacuse the spring elements from the individual oscillators overlap significantly. On the other hand, in Fig. 6(b) the spring elements overlap less because $\varepsilon$ is somewhat smaller. Finally, in Fig. 6(c) the spatial memory approaches zero as $\varepsilon \rightarrow 0$, and the spatial stiffness density approaches the stiffness of simple discrete springs.

\section{Derivation of the boundary impedance of the $n$th set of oscillators}

The vibration of the master results in a force at the interface between master and attached fuzzy; this action on the fuzzy we denote the contact force. Now, from the vibration velocity $\underline{v}(x)$ along the fuzzy connection boundary one can express the total contact force $F_{-, n}^{\prime}\left(x_{0}\right)$ per unit length at $x_{0}$ due to the $n$th set of oscillators as

$$
\underline{F}_{\varepsilon, n}^{\prime}\left(x_{0}\right)=\int_{L_{\text {fuzzy }}} \underline{z}_{\varepsilon, n}\left(x_{0}-x\right) \underline{v}(x) d x,
$$

where $z_{\varepsilon, n}\left(x_{0}-x\right)$ is the boundary impedance associated with the $n$th set of oscillators. This impedance depends only on the difference $\left(x_{0}-x\right)$, so that $\underline{z}_{\varepsilon, n}\left(x_{0}-x\right)=\underline{z}_{\varepsilon, n}\left(x-x_{0}\right)$ in analogy to the stiffness distribution function in Eq. (7). Although an expression for $z_{\varepsilon, n}\left(x_{0}-x\right)$ is shown in Ref. 3 , it has not been derived in the open literature. The authors believe that such a derivation is essential in order to appreciate and understand the characteristics of a fuzzy with spatial memory. It is also anticipated that such a derivation will ease the usabil-

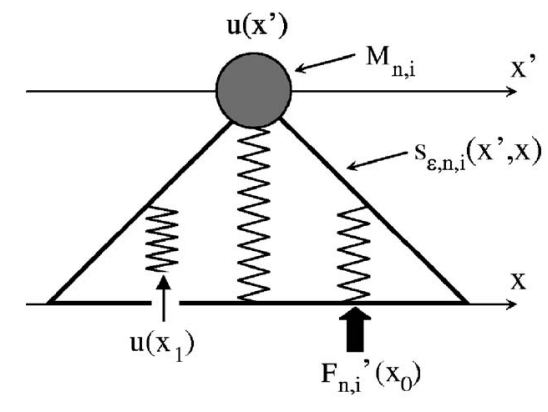

(a)

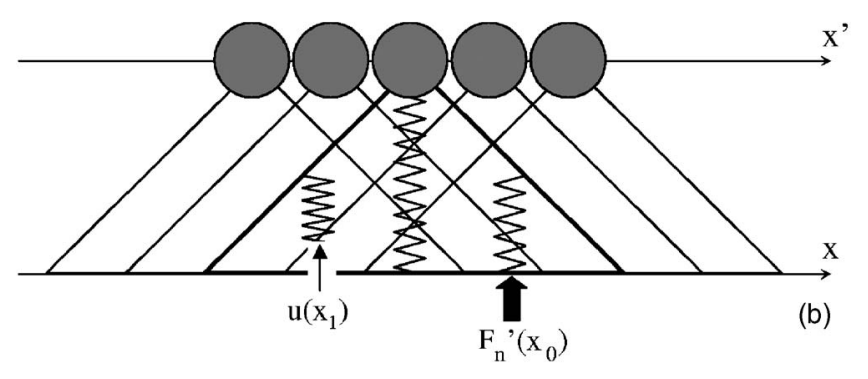

FIG. 7. Derivation of the $n$th contribution to the fuzzy boundary impedance. (a) Boundary impedance $z_{\varepsilon, n, i}\left(x_{0}-x_{1}\right)$ of the $i$ th oscillator of the $n$th set of spatial oscillators. (b) Boundary impedance $z_{\varepsilon, n}\left(x_{0}-x_{1}\right)$ of the $n$th set of spatial oscillators.

ity of Soize's theory considerably. In view of this, a step by step derivation of $F_{\varepsilon, n}^{\prime}\left(x_{0}\right)$ will be presented in what follows.

Once again, only one spatial oscillator is considered, say, the $i$ th of the $n$th set of oscillators. This oscillator is sketched in Fig. 7(a). For this particular oscillator we first seek an expression for the boundary impedance $\underline{z}_{\varepsilon, n, i}\left(x_{0}\right.$ $-x_{1}$ ) with a spatial contribution over the differential length $d x$ is defined as

$$
\underline{F}_{n, i}^{\prime}\left(x_{0}\right)=\left.\underline{z}_{\varepsilon, n, i}\left(x_{0}-x_{1}\right) \underline{v}\left(x_{1}\right) d x\right|_{\underline{\underline{v}}\left(x \notin\left[x_{1}, x_{1}+d x\right]\right)=0},
$$

where $\underline{F}_{n, i}^{\prime}\left(x_{0}\right)$ is the force per unit length that excites the connection boundary at $x_{0}$ and $\underline{v}\left(x_{1}\right)=i \omega \underline{u}\left(x_{1}\right)$ is the base velocity of the spring element at $x_{1}$. Now, the spring element at $x_{1}$ is given a displacement $\underline{u}\left(x_{1}\right)$ at its base, whereas all other springs elements are locked such that $\underline{u}\left(x \neq x_{1}\right)=0$, see Fig. 7(a). As the mass $M_{n, i}$ undergoes a displacement $\underline{u}\left(x^{\prime}\right)$ the induced spring force $\underline{F}_{S, n, i}\left(x_{1}\right)$ in a differential neighborhood $d x$ around $x_{1}$ becomes

$$
\underline{F}_{S, n, i}\left(x_{1}\right)=\underline{s}_{\varepsilon, n, i}\left(x^{\prime}-x_{1}\right)\left(\underline{u}\left(x_{1}\right)-\underline{u}\left(x^{\prime}\right)\right) d x .
$$

Due to the motion of the mass, a reaction force $\underline{F}_{M, n, i}\left(x^{\prime}\right)$ influences the spring element and this force is given as

$$
\underline{F}_{M, n, i}\left(x^{\prime}\right)=-\omega^{2} M_{n, i} \underline{u}\left(x^{\prime}\right) .
$$

Additionally, the motion of the mass also introduces forces in the remaining spring elements, and their total spring force $\underline{F}_{S, n, i}\left(x \neq x_{1}\right)$ can be found as

$$
\begin{gathered}
\underline{F}_{S, n, i}\left(x \neq x_{1}\right)=-\underline{s}_{\varepsilon, n, i}\left(x^{\prime}-x_{1}\right) \underline{u}\left(x^{\prime}\right) d x+\int_{L_{\mathrm{fuzzy}}} \underline{s}_{\varepsilon, n, i}\left(x^{\prime}\right. \\
-x) \underline{u}\left(x^{\prime}\right) d x=-\underline{s}_{\varepsilon, n, i}\left(x^{\prime}-x_{1}\right) \underline{u}\left(x^{\prime}\right) d x+\underline{s}_{n, i},
\end{gathered}
$$

where $\underline{s}_{n, i}$ is the total stiffness of the spring elements of the oscillator. Because of force equilibrium the sum of the spring

L. Friis and M. Ohlrich: Structural fuzzy with continuous boundary 
force $\underline{F}_{S, n, i}\left(x \neq x_{1}\right)$ and the reaction force of the mass $\underline{F}_{M, n, i}\left(x^{\prime}\right)$ is equal to the spring force $\underline{F}_{S, n, i}\left(x_{1}\right)$ at $x_{1}$. Thus, by combination of Eqs. (10)-(12) we get

$$
\begin{aligned}
& \underline{F}_{S, n, i}\left(x_{1}\right)=\underline{F}_{M, n, i}\left(x^{\prime}\right)+\underline{F}_{S, n, i}\left(x \neq x_{1}\right), \\
& \underline{s}_{\varepsilon, n, i}\left(x^{\prime}-x_{1}\right)\left(\underline{u}\left(x_{1}\right)-\underline{u}\left(x^{\prime}\right)\right) d x \\
& \quad=\left(-\underline{s}_{\varepsilon, n, i}\left(x^{\prime}-x_{1}\right) d x+\underline{s}_{n, i}-\omega^{2} M_{n, i}\right) \underline{u}\left(x^{\prime}\right),
\end{aligned}
$$

Rearranging Eq. (13) we find an expression for $\underline{u}\left(x^{\prime}\right)$ as a function of $\underline{u}\left(x_{1}\right)$, which yields

$$
\underline{u}\left(x^{\prime}\right)=\frac{\underline{s}_{\varepsilon, n, i}\left(x^{\prime}-x_{1}\right) \underline{u}\left(x_{1}\right) d x}{-\omega^{2} M_{n, i}+\underline{s}_{n, i}} .
$$

Further, the force per unit length $\underline{F}_{n, i}^{\prime}\left(x_{0}\right)$ at $x_{0}$ at the connection boundary is given as

$$
\underline{F}_{n, i}^{\prime}\left(x_{0}\right)=\underline{s}_{\varepsilon, n, i}\left(x^{\prime}-x_{0}\right)\left(\underline{u}\left(x_{0}\right) \delta_{x_{0}, x_{1}}-\underline{u}\left(x^{\prime}\right)\right),
$$

where $\delta_{x_{0}, x_{1}}$ is the Kronecker delta, that is, $\delta_{x_{0}, x_{1}}=1$ when $x_{0}=x_{1}$, and otherwise zero. Finally, if Eq. (14) is substituted in Eq. (15) we get the force per unit length $\underline{F}_{n, i}^{\prime}\left(x_{0}\right)$ exerted onto the fuzzy connection boundary due to its displacement $\underline{u}\left(x_{1}\right)$ :

$$
\begin{aligned}
\underline{F}_{n, i}^{\prime}\left(x_{0}\right) & =\underline{s}_{\varepsilon, n, i}\left(x^{\prime}-x_{0}\right)\left(\delta_{x_{0}, x_{1}} \underline{u}\left(x_{0}\right)-\frac{\underline{s}_{\varepsilon, n, i}\left(x^{\prime}-x_{1}\right) \underline{u}\left(x_{1}\right) d x}{-\omega^{2} M_{n, i}+\underline{s}_{n, i}}\right) \\
& =\underline{s}_{\varepsilon, n, i}\left(x^{\prime}-x_{0}\right)\left(\delta_{x_{0}, x_{1}}-\frac{\underline{s}_{\varepsilon, n, i}\left(x^{\prime}-x_{1}\right) d x}{-\omega^{2} M_{n, i}+\underline{s}_{n, i}}\right) \underline{u}\left(x_{1}\right) .
\end{aligned}
$$

According to Eq. (16) $\underline{z}_{\varepsilon, n, i}\left(x_{0}-x_{1}\right) d x$ reads

$$
\begin{aligned}
\underline{z}_{\varepsilon, n, i}\left(x_{0}-x_{1}\right) d x= & \frac{\underline{s}_{\varepsilon, n, i}\left(x^{\prime}-x_{0}\right)}{i 2 \pi f}\left(\delta_{x_{0}, x_{1}}\right. \\
& \left.-\frac{\underline{S}_{\varepsilon, n, i}\left(x^{\prime}-x_{1}\right) d x}{-\omega^{2} M_{n, i}+\underline{s}_{n, i}}\right) .
\end{aligned}
$$

Now, this is only the impedance of the $i$ th oscillator of the $n$th set. Assume again that there is an infinite number of identical oscillators overlapping one another on the fuzzy connection boundary [see Fig. 7(b)], such that a mass element $M_{n, i}$ is located at each of all positions along the $x^{\prime}$ axis. Further, each of these oscillators has a total stiffness $\underline{s}_{n, i}$. We now seek an expression for the impedance $z_{\varepsilon, n}\left(x_{0}-x_{1}\right)$ of the $n$th set of oscillators. First the oscillators are given the displacement $\underline{u}\left(x_{1}\right)$ at position $x_{1}$, whereas all other positions at their spring bases are locked. Then the total force per unit length $F_{n}^{\prime}\left(x_{0}\right)$ at $x_{0}$ is found by integrating the expression in Eq. (16) with respect to $x^{\prime}$, and this gives

$$
\begin{aligned}
\underline{F}_{n}^{\prime}\left(x_{0}\right)= & \int_{L_{\mathrm{fuzzy}}} \underline{s}_{\varepsilon, n, i}\left(x^{\prime}-x_{0}\right)\left(\delta_{x_{0}, x_{1}}\right. \\
& \left.-\frac{\underline{S}_{\varepsilon, n, i}\left(x^{\prime}-x_{1}\right) d x}{-\omega^{2} M_{n, i}+\underline{s}_{n, i}}\right) \underline{u}\left(x_{1}\right) d x^{\prime} \\
= & \left(\int_{L_{\mathrm{fuzzy}}} \underline{s}_{\varepsilon, n, i}\left(x^{\prime}-x_{0}\right) \delta_{x_{0}, x_{1}} d x^{\prime}\right. \\
& \left.-\int_{L_{\mathrm{fuzzy}}} \frac{\underline{s}_{\varepsilon, n, i}\left(x^{\prime}-x_{0}\right) \underline{s}_{\varepsilon, n, i}\left(x^{\prime}-x_{1}\right) d x}{-\omega^{2} M_{n, i}+\underline{s}_{n, i}}\right) \underline{u}\left(x_{1}\right)
\end{aligned}
$$

J. Acoust. Soc. Am., Vol. 123, No. 2, February 2008

$$
\begin{aligned}
= & \left(\underline{s}_{n, i} \delta_{x_{0}, x_{1}}\right. \\
& \left.-\int_{L_{\mathrm{fuzzy}}} \frac{\underline{s}_{n, i}^{2} g\left(x^{\prime}-x\right) g\left(x^{\prime}-x_{0}\right) d x}{-\omega^{2} M_{n, i}+\underline{s}_{n, i}} d x^{\prime}\right) \underline{u}\left(x_{1}\right) \\
= & \left(\underline{s}_{n, i} \delta_{x_{0}, x_{1}}-\frac{\underline{s}_{n, i}^{2} d x}{-\omega^{2} M_{n, i}+\underline{s}_{n, i}} \int_{L_{\mathrm{fuzzy}}} g\left(x^{\prime}-x_{1}\right) g\left(x^{\prime}\right.\right. \\
& \left.\left.-x_{0}\right) d x^{\prime}\right) \underline{u}\left(x_{1}\right) .
\end{aligned}
$$

The integration in the last line of Eq. (18) can be recognized as the convolution product $(g * g)\left(x_{0}-x_{1}\right)$ and the expression can therefore be simplified to

$$
\begin{aligned}
\underline{F}_{n}^{\prime}\left(x_{0}\right)= & \underline{s}_{n, i}\left(\delta_{x_{0}, x_{1}}-\frac{\underline{s}_{n, i}}{-\omega^{2} M_{n, i}+\underline{s}_{n, i}}(g * g)\left(x_{0}\right.\right. \\
& \left.\left.-x_{1}\right) d x\right) \underline{u}\left(x_{1}\right) .
\end{aligned}
$$

Finally, by substituting $\underline{s}_{n, i}=\omega_{n}^{2} M_{n, i}(1+i \eta)$ and $\underline{v}(x)$ $=i 2 \pi \underline{f} \underline{u}(x)$ in Eq. (19) we have that

$$
\begin{aligned}
\underline{F}_{n}^{\prime}\left(x_{0}\right)= & -i 2 \pi f\left(\frac{f_{r, n}^{2}}{f^{2}}\right)(1+i \eta) M_{n, i}\left(\delta_{x_{0}, x_{1}}\right. \\
& \left.-\frac{f_{r, n}^{2}(1+i \eta)}{f_{r, n}^{2}(1+i \eta)-f^{2}}(g * g)\left(x_{0}-x_{1}\right) d x\right) \underline{v}\left(x_{1}\right) .
\end{aligned}
$$

Hence, the total impedance $\underline{z}_{\varepsilon, n}\left(x_{0}-x_{1}\right)$ of the $n$th set of oscillators reads

$$
\begin{aligned}
\underline{z}_{\varepsilon, n}\left(x_{0}-x_{1}\right) d x= & -i 2 \pi f\left(\frac{f_{r, n}^{2}}{f^{2}}\right)(1+i \eta) M_{n, i}\left(\delta_{x_{0}, x_{1}}\right. \\
& \left.-\frac{f_{r, n}^{2}(1+i \eta)}{f_{r, n}^{2}(1+i \eta)-f^{2}}(g * g)\left(x_{0}-x_{1}\right) d x\right) .
\end{aligned}
$$

A structural fuzzy composed of $N$ sets of infinitely many identical oscillators as described earlier is homogenous, as the boundary impedance only depends on the distance $\left|x_{0}-x_{1}\right|$. Further, if $\varepsilon \rightarrow 0$, then Eq. (21) reduces to the boundary impedance of infinitely many identical simple oscillators as illustrated in Fig. 6(c). Examining the expression in Eq. (21), it is seen that the transfer impedances are proportional to the convolution product $(g * g)\left(x_{0}-x_{1}\right)$, as the term $\delta_{x_{0}, x_{1}}$ is zero when $x_{0} \neq x$. Figure 8 shows this convolution and it is seen that the transfer impedances extends a distance of $2 \varepsilon$ to each side of $x$. This means that the actual spatial memory is $4 \varepsilon$, and that the transfer impedances are zero for $\left|x_{0}-x\right|$ $\geqslant 2 \varepsilon$. Note that the areas below $g\left(x_{0}-x\right)$ and $\left(g^{*} g\right)\left(x_{0}-x_{1}\right)$ are both equal to 1 .

\section{Soize's local equivalent oscillator}

A numerical implementation of the boundary impedance $z_{\varepsilon, n}$ in Eq. (21) is unfortunately rather complicated due to its nonlocal nature. This requires for instance the use of a finite 


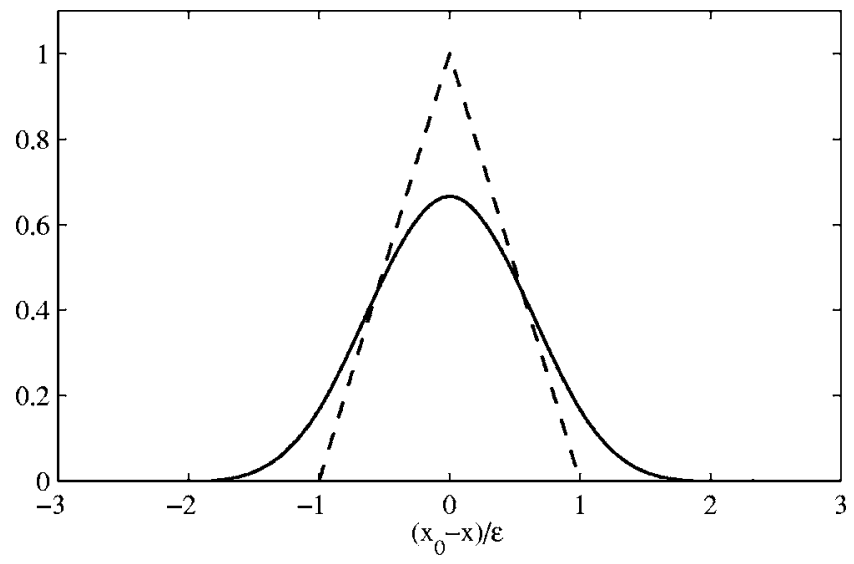

FIG. 8. Examination of the magnitude distribution of the transfer impedance. Functions: ---, $g\left(x_{0}-x\right) /(1 / \varepsilon) ;-,\left(g^{*} g\right)\left(x_{0}-x\right) /(1 / \varepsilon)$.

element model with special fuzzy elements. As mentioned earlier, the main purpose of the fuzzy structure theory is to serve as a simple modeling tool. Therefore, Soize introduced the equivalent local oscillator. The idea is that the $n$th set of infinitely many identical equivalent oscillators, can replace the $n$th set of spatial oscillators. This also implies that the contact force per unit length $\underline{F}_{\text {equ }, n}^{\prime}\left(x_{0}\right)$ introduced by the local equivalent oscillator at $x_{0}$ must be equal to the contact force $F_{\varepsilon, n}^{\prime}\left(x_{0}\right)$ given in Eq. (8). This is achieved by introducing the so-called equivalent coupling factor $\alpha$, which transforms all the nonlocal force contributions of the spatial oscillators into equivalent local contributions. As one can imagine, $\alpha$ generally varies with frequency and is both dependent on the length of the spatial memory $2 \varepsilon$ and the motion of the master $u(x)$.

Now, let us assume that the $n$th set of equivalent oscillators consist of an infinite number of identical oscillators distributed on the fuzzy connection boundary. Further, each equivalent oscillator has a mass $M_{n, i}$, and masses are located at all positions along the $x^{\prime}$ axis. For the $n$th set of equivalent oscillators the boundary impedance $\underline{z}_{\text {equ }, n}\left(x_{0}\right)=\underline{F}_{n}^{\prime}\left(x_{0}\right) / \underline{v}\left(x_{0}\right)$ is given as ${ }^{6}$

$$
\begin{aligned}
\underline{z}_{\mathrm{equ}, n}\left(x_{0}\right)= & \frac{\underline{S}_{n, i}}{i \omega}\left(1-\frac{\underline{s}_{n, i}}{\underline{s}_{n, i}-\omega^{2} M_{n, i}} \alpha\right)=i 2 \pi f M_{n, i} \\
& \times\left(\frac{f_{r}^{2}(1+i \eta)\left(1-\left(f_{r} / f\right)^{2}(1+i \eta)(1-\alpha)\right)}{f^{2}-f_{r}^{2}(1+i \eta)}\right),
\end{aligned}
$$

where $\alpha \in] 0,1]$. If $\alpha$ is chosen properly then the boundary impedance $\underline{z}_{\mathrm{equ}, n}\left(x_{0}\right)$ in Eq. (22) can replace successfully the boundary impedance $z_{\varepsilon, n}\left(x_{0}-x_{1}\right)$ of the $n$th set of spatial oscillators $^{6}$ as was given in Eq. (21). The frequency variation of this equivalent impedance $\underline{z}_{\text {equ }, n}\left(x_{0}\right)$ is shown in Fig. 9 for different values of $\alpha$. As indicated in Fig. 9, we now suggest that the equivalent oscillator can be interpreted as a simple oscillator with spring stiffness $\underline{s}_{1}$ where the mass has been grounded by a second spring with stiffness $\underline{s}_{2}$. It applies that $\underline{s}_{n, i}=\underline{s}_{1}+\underline{s}_{2}$ and the mass of the grounded oscillator is $M_{n, i}$. The relationship between the impedance in Eq. (22) and the impedance of the grounded oscillator $z_{\text {ground, } n}$ in Fig. 9 is $\underline{z}_{\text {equ }, n}=\underline{z}_{\text {ground, }, n} / \alpha$ where $\alpha=\underline{s}_{1} /\left(\underline{s}_{1}+\underline{s}_{2}\right)$. Note that $\alpha \rightarrow 1$ when
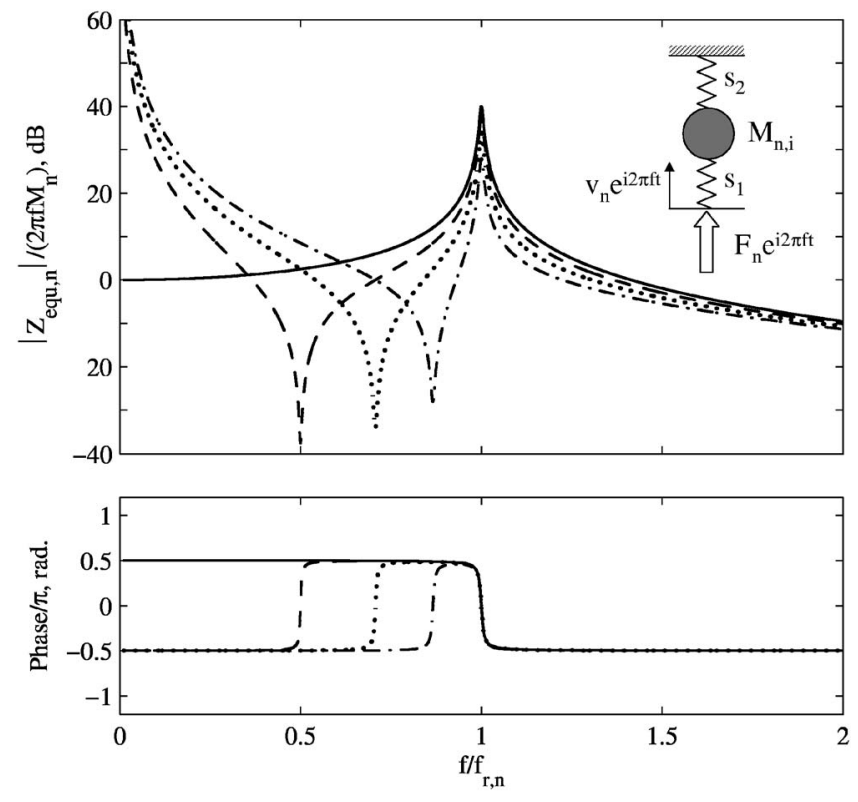

FIG. 9. Frequency variation of normalized impedance, $\underline{Z}_{\text {equ, } n} /\left(2 \pi f M_{n}\right)$ for different values of the equivalent coupling factor $\alpha:-, 1 ;---, 0.75 ; \cdots, 0.5$; $-\cdot-, 0.25$.

$\underline{s}_{2} \rightarrow 0$ and $\underline{z}_{\mathrm{equ}, n}$ will then approach the impedance of a simple oscillator, Eq. (1). Also, when $\alpha \neq 1$ the oscillator is stiffness controlled at low frequencies. It should be mentioned that the impedance of a set of spatial oscillators results in a stiffness-controlled behaviour of the master at low frequencies. The reason is that the mass-less bar supporting the point mass in Fig. 5(a) is restricted to translational motion and therefore unable to rotate. Any rotation of the master at low frequencies is therefore restricted by the springs.

\section{E. The equivalent coupling factor}

Soize states that it is not self-evident that the local equivalent oscillator can model correctly a structural fuzzy with spatial memory. ${ }^{6}$ And as one can imagine, $\alpha$ has to be chosen carefully. Finding a relationship between $\varepsilon$ and $\alpha$ requires matching of boundary forces using the impedances found in Eqs. (21) and (22), frequency by frequency. Such results have been published by Soize ${ }^{6}$ and they show $\alpha$ as a function of the spatial memory $2 \varepsilon$ for different frequency bands for a simply supported beam. The authors of the present article, however, suggest that $\alpha$ should be determined in a more general way as a function of the ratio $\varepsilon / \lambda$ where $\lambda$ is the free wavelength in the master, which here is restricted to undergo one-dimensional wave motion only. By transforming Soize's data, it is revealed that a unique relationship between $\alpha$ and $\varepsilon / \lambda$ is found. The transformed data of $\alpha$ as a function of $\varepsilon / \lambda$ are shown in Fig. 10; these results have been fitted with a fourth-order polynomial. It should be noted that the free wavelength is defined only for sinusoidal variations, and for structures with more complicated eigenfunctions, we therefore suggest substituting $\lambda$ with twice the distance between adjacent nodes.

Finally, it should be mentioned that a general and simple method of predicting $\alpha$ has been the subject of the authors' 


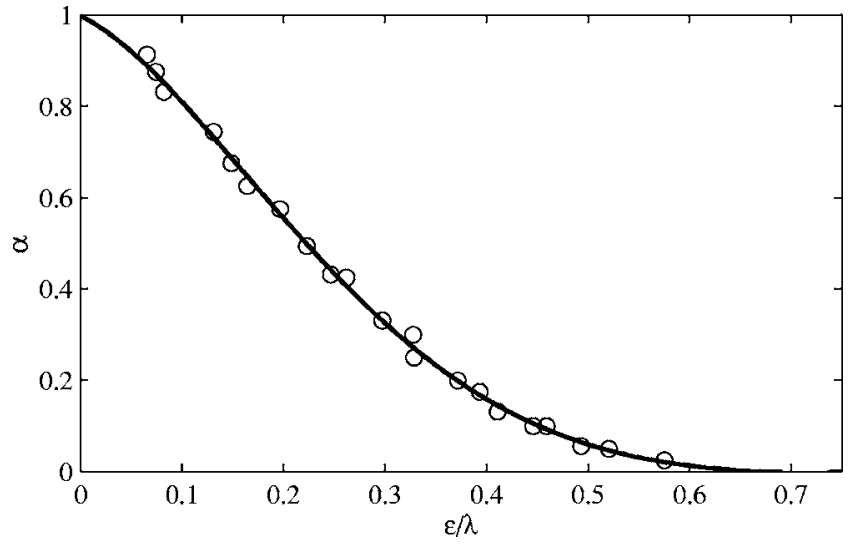

FIG. 10. Variation of the equivalent coupling factor $\alpha$ with $\varepsilon / \lambda:(\bigcirc)$, data computed from read-off results in Ref. 6; - , polynomial fit.

latest work and will shortly be submitted for publication together with a practical validation of the associated equivalent modelling method.

\section{SMOOTHED EXPRESSION FOR THE BOUNDARY IMPEDANCE OF STRUCTURAL FUZZY WITH SPATIAL MEMORY}

The fuzzy structure theory developed by Soize was originally intended for finite element modeling. To determine the damping induced in the master, Soize developed his own methods based on probabilistic concepts in order to account for model uncertainties. A new proposition for a simplified deterministic method for predicting the mean damping induced by structural fuzzy with spatial memory is presented in the following. The purpose of this model is to illustrate the main effects of including spatial memory in the modeling of structural fuzzy.

As a starting point we consider a structural fuzzy consisting of $N$ sets of spatial oscillators with different natural frequencies. The total boundary impedance of the structural fuzzy can be determined as the sum of the impedance contributions from these $N$ sets for which the impedance of the $n$th set, $n \in[1, N]$, was presented in Eq. (21). The total boundary impedance $\underline{z}_{\text {fuzzy, } \varepsilon}\left(x_{0}-x_{1}\right)$ thus becomes

$$
\underline{z}_{\text {fuzzy }, \varepsilon}\left(x_{0}-x_{1}\right)=\sum_{n=1}^{N} \underline{z}_{\varepsilon, n}\left(x_{0}-x_{1}\right) \text {. }
$$

So far, only Soize has presented a method of predicting the boundary impedance of fuzzy with spatial memory. ${ }^{6}$ Now, applying the same approach as in Sec. II a deterministic expression for the boundary impedance of the structural fuzzy with spatial memory can be found. Let us approximate the expression in Eq. (23) by infinitely many sets of spatial oscillators resonating between $f_{r \text {,lower }}$ and $f_{r \text {,upper }}$. Hereby the general expression for the total fuzzy boundary impedance attached at area $A$ of the master becomes

$$
\underline{z}_{\text {fuzzy }, \varepsilon}\left(x_{0}-x_{1}\right)=\int_{f_{r, \text { lower }}}^{f_{r, \text { upper }}} z_{\varepsilon}\left(x_{0}-x_{1}, f_{r}\right) d f_{r},
$$

or, by inserting the expression from Eq. (21) we get

$$
\begin{aligned}
\underline{z}_{\text {fuzzy }, \varepsilon}\left(x_{0}-x_{1}\right) d x= & -\frac{i 2 \pi f}{A} \int_{f_{r, \text { lower }}}^{f_{r, \text { upper }}}\left(\frac{f_{r}^{2}}{f^{2}}\right)(1+i \eta) m_{\text {fuzzy }}\left(f_{r}\right) \\
& \times\left(\delta_{x_{0}, x_{1}}-\frac{f_{r}^{2}(1+i \eta)}{f_{r}^{2}(1+i \eta)-f^{2}}\right. \\
& \left.\times(g * g)\left(x_{0}-x_{1}\right) d x\right) d f_{r}
\end{aligned}
$$

where $m_{\text {fuzzy }}\left(f_{r}\right) d f_{r}$ again represents the total mass of the fuzzy resonating between the frequencies $f_{r}$ and $f_{r}+d f_{r}$. Moreover, the same simple approach can be applied to find the equivalent boundary impedance $\underline{z}_{\text {fuzzy, equ }}\left(x_{0}\right)$ if $M_{n}$ is replaced by $m_{\text {fuzzy }}\left(f_{r}\right) d f_{r}$. A smoothed version of the equivalent boundary impedance then yields

$$
\begin{aligned}
\underline{z}_{\text {fuzzy } \text { equ }}\left(x_{0}-x_{1}\right)= & \int_{f_{r, \text { lower }}}^{f_{r \text {,upper }}} z_{\text {equ }}\left(x_{0}, f_{r}\right) d f_{r} \\
= & -\frac{i 2 \pi f}{A} \int_{f_{r, \text { lower }}}^{f_{r, \text { upper }}}\left(\frac{f_{r}^{2}}{f^{2}}\right)(1+i \eta) m_{\text {fuzzy }}\left(f_{r}\right) \\
& \times\left(1-\frac{f_{r}^{2}(1+i \eta)}{f_{r}^{2}(1+i \eta)-f^{2}} \alpha\right) d f_{r}
\end{aligned}
$$

It should be noted that the equivalent coupling factor $\alpha$ generally is a function of frequency. Nevertheless, according to Fig. 10 it is seen that $\alpha$ is constant for a specific value of $\varepsilon / \lambda$. With the new expression for the boundary impedance of structural fuzzy with spatial memory in Eq. (25) it is therefore possible to model and examine the effects of structural fuzzy with spatial memory in a simple way. For simple cases of mass distributions $m_{\text {fuzzy }}\left(f_{r}\right)$ the integration can be done analytically, whereas the use of more realistic mass distributions will require a numerical integration.

\section{BEAM MASTER STRUCTURE WITH STRUCTURAL FUZZY}

The influence of structural fuzzy with and without spatial memory will now be illustrated by a numerical example. The finite element method $^{13}$ is being used for solving the harmonically forced vibration response of a simply supported Bernoulli-Euler beam, which is considered as the master structure. A fuzzy substructure is attached on the whole length $L$ of the beam, so that $L_{\text {fuzzy }}=L$. The damping loss factor of the beam is 0.005 and the loss factor of the fuzzy oscillator springs is 0.03 . The resonating mass per unit frequency, $m_{\text {fuzzy }}\left(f_{r}\right)$, is taken to follow a normal distribution, giving

$$
m_{\text {fuzzy }}\left(f_{r}\right)=\frac{M_{\text {fuzzy }}}{\operatorname{std} \sqrt{2 \pi}} e^{-\left(f_{r 0}-f_{r}\right)^{2} /\left(2 \cdot \mathrm{std}^{2}\right)},
$$

where $f_{r 0}$ is the center frequency and std is the standard deviation. This chosen mass distribution is shown in Fig. 11 as a function of the beam's nondimensional frequency $\Omega$ being defined as 


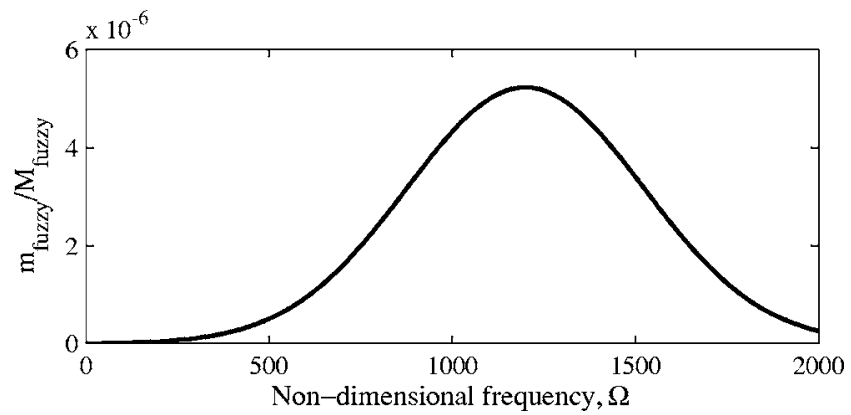

FIG. 11. Normalized resonating mass per unit frequency described by a normal distribution with a centre frequency $f_{r 0}$ corresponding to $\Omega=1200$ and a standard deviation std of $\Omega=750$.

$$
\Omega=2 \pi f \sqrt{\frac{12 \rho}{E}} \frac{L^{2}}{h},
$$

where $h$ is the beam thickness, and $\rho$ and $E$ is the density and Young's modulus of the beam material, respectively. For this distribution the bounding frequencies $f_{r \text {,lower }}$ and $f_{r \text {,upper }}$ correspond to $\Omega=0$ and $\Omega=\infty$, respectively. The center frequency $f_{r 0}$ corresponds to $\Omega=1200$ and the standard deviation std is $\Omega=750$. Moreover, the total mass of the fuzzy $M_{\text {fuzzy }}$ is taken to be one-twentieth of the beam mass, $\rho S L$, where $S$ is its cross-sectional area.

The boundary impedance of the fuzzy, Eq. (25), is computed by numerical integration; it is assumed that the equivalent coupling factor $\alpha$ is constant with frequency, which means that the ratio $\varepsilon / \lambda$ is constant, whereas $\varepsilon$ and $\lambda$ decrease with frequency at the same rate. Figure 12 shows computed results of the fuzzy boundary impedance $\underline{z}_{\text {fuzzy,equ }}\left(x_{0}\right)$ as a function of the nondimensional frequency for different values of $\alpha$.
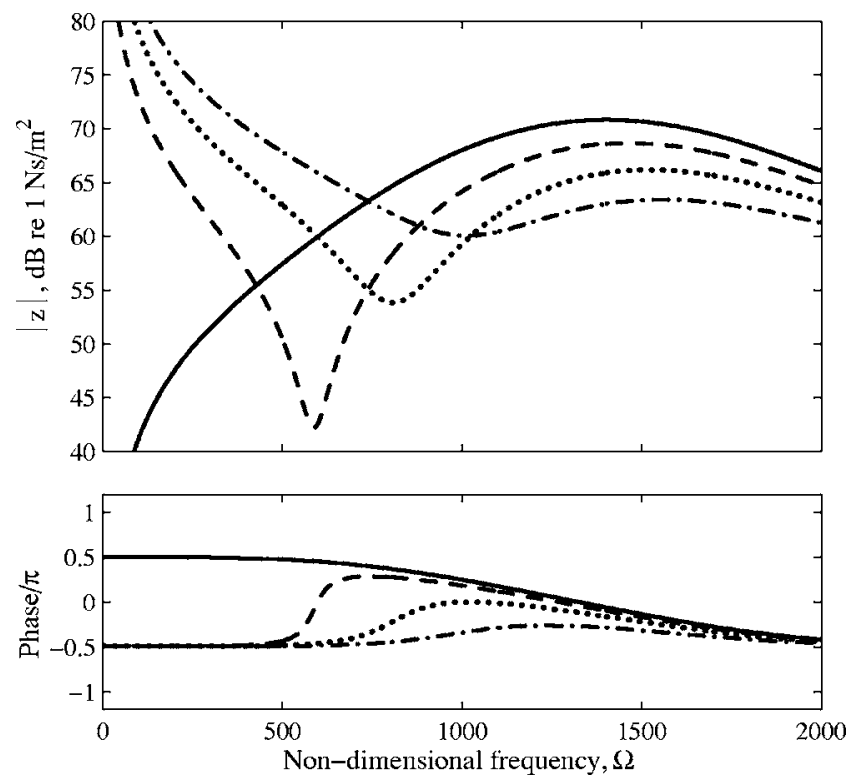

FIG. 12. Amplitude and phase of the fuzzy boundary impedance $\underline{z}_{\text {fuzzy,equ }}$ The total mass of the fuzzy is $1 / 20$ of the master and has a normaldistributed resonating mass per unit frequency. Results are shown for different values of the equivalent coupling factor $\alpha$ : -, $1 ;---, 0.75 ; \cdots, 0.5 ;---$, 0.25 .
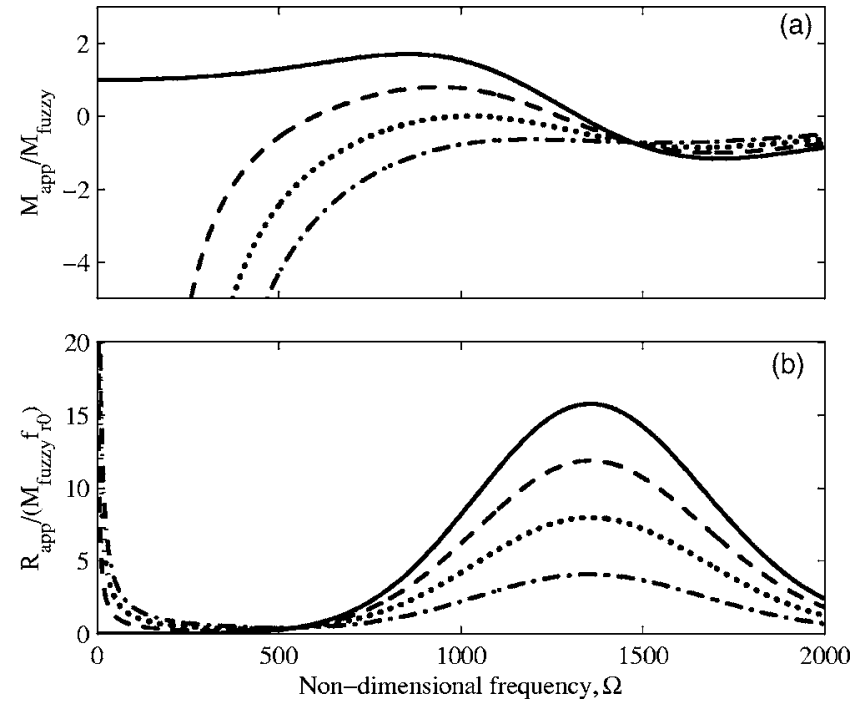

FIG. 13. (a) Normalized apparent mass, $M_{\text {app }}=\operatorname{Im}\left(\underline{z}_{\text {fuzzy,equ }}\right) / \omega$, and (b) normalized apparent damping, $R_{\text {app }}=\operatorname{Re}\left(\underline{z}_{\text {fuzzy,equ }}\right)$, of the fuzzy boundary impedance for different values of the $\alpha:-, 1 ;---, 0.75 ; \cdots, 0.5 ;---, 0.25$.

From these results a number of observations can be made. First, it is clearly seen that the structural fuzzy without memory is mass-controlled at frequencies below $\Omega=500$, as the amplitude slope of the boundary impedance is positive and the phase equals $\pi / 2$. This is not the case for structural fuzzy with memory, which is clearly spring-like with a negative amplitude slope and a phase of $-\pi / 2$; this will always be the case when $\alpha<1$. Second, between $\Omega=500$ and $\Omega$ $=2000$ the phase of the fuzzy without memory changes smoothly from $\pi / 2$ to approximately $-\pi / 2$. During this change the real part of the impedance exhibits high values and the fuzzy therefore has a high damping effect. The impedance for $\alpha=0.75$ has a sudden phase change of $\pi$ after $\Omega=500$ and hereafter it closely follows the phase of the fuzzy for $\alpha=1$. Examining the other two cases of smaller $\alpha$ values, it is found that the phase change is less pronounced and occurs at a higher frequency; therefore the fuzzy never reaches true mass-like behavior. It is also seen that the amplitude of the impedance becomes significantly lower when $\alpha$ is decreased, and this results in a weakening of the effect of the fuzzy. This is clearly observed for frequencies above $\Omega=1000$.

Inspired by Pierce et $a .^{8}$ the behavior and actual damping effect of the fuzzy is conveniently demonstrated by examining the corresponding apparent mass, $M_{\text {app }}(f)$ $=\operatorname{Im}\left(\underline{z}_{\text {fuzzy,equ }}\right) /(2 \pi f)$, and apparent damping $R_{\text {app }}(f)$ $=\operatorname{Re}\left(\underline{z}_{\text {fuzzy,equ }}\right)$. Both quantities are shown in normalized form in Fig. 13 for different values of $\alpha$. Figure 13(a) shows that the apparent mass of the fuzzy without memory is equal to the total mass of the fuzzy at $\Omega=0$, and up to around $\Omega$ $=1170$ the apparent mass is higher than the total mass. Further, around $\Omega=1340$ the quantity becomes negative, which indicates a spring-controlled behavior. The three cases of fuzzy with memory clearly differ from this behaviour by being mostly spring-like in the whole frequency range. Above, say $\Omega=1350$, the apparent mass is very close in all cases. The apparent damping is plotted in Fig. 13(b), and it is seen that the damping effect of the fuzzy decreases significantly

L. Friis and M. Ohlrich: Structural fuzzy with continuous boundary 

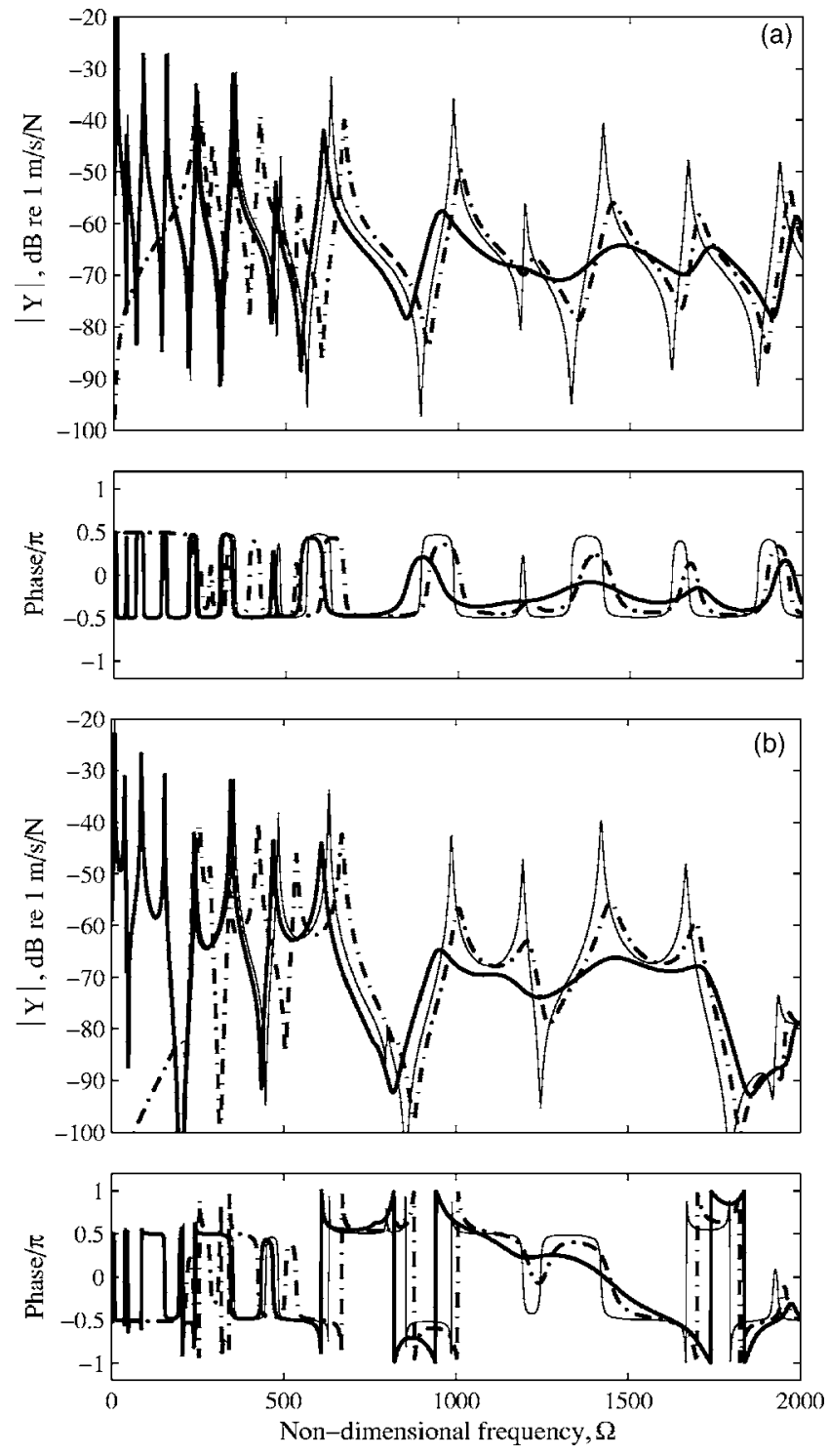

FIG. 14. Vibration velocity response per unit harmonic force, $\underline{Y}\left(x, x_{0}\right)$ $=\underline{v}(x) / \underline{F}\left(x_{0}\right)$, at (a) $x=0.445 L$ and (b) $x=0.785 L$ of a simply supported beam excited at $x_{0}=0.445 L$. Condition: - without structural fuzzy and with structural fuzzy for $\alpha$ : - $1 ;----, 0.75 ; \cdots, 0.5 ;-\cdot-, 0.25$.

when $\alpha$ is reduced. The reason for this is that a reduction of $\alpha$ corresponds to an increase of $\varepsilon$. Due to the rotational motion in the master, the spring elements in the spatial oscillators counteract one another when $\varepsilon>0$. This also implies that the impedance of the structural fuzzy is reduced significantly, and this results in a lower dissipation induced in the master. For the chosen mass distribution, the maximum damping effect occurs around $\Omega=1360$, which is higher than the center frequency of $\Omega=1200$. At low frequencies below $\Omega=100$ it is seen that the apparent damping becomes very high. This nonphysical behavior is caused by the approximate modeling of the fuzzy boundary impedance, which goes toward infinity at $\Omega=0$.

Finally, Fig. 14 shows results for the vibration velocity response of the simply supported master beam, without and with the fuzzy substructure, which was discussed earlier. The vibration responses at $x=0.445 \mathrm{~L}$ and $x=0.785 \mathrm{~L}$ are given in terms of the beam's mobility $\underline{Y}\left(x, x_{0}\right)=\underline{v}(x) / \underline{F}\left(x_{0}\right)$ for harmonic force excitation at $x_{0}=0.445 \mathrm{~L}$. Considering both Figs. 14(a) and 13(b) it is seen that the fuzzy without spatial memory, $\alpha=1$, introduces a high damping in the master beam. The effect of this stretches over a relatively wide frequency band, which covers at least six flexural modes of the beam. From around $\Omega=950$ to $\Omega=1740$ the damping effect is very pronounced and the vibration velocity of the master is dampened by up to $25 \mathrm{~dB}$. Further, this structural fuzzy, causes the resonances of the beam structure to shift downwards into the region where the fuzzy is mass-like. This is the case in the range from $\Omega=0$ to $\Omega=1340$. Above $\Omega$ $=1340$ the fuzzy becomes spring controlled and the resonances are shifted upwards.

Next, considering the case of structural fuzzy with a spatial memory of $\alpha=0.25$, it is evident that the spatial memory significantly decreases the damping effect of the fuzzy. In the main damping region of the fuzzy, the damping effect is reduced by almost $10 \mathrm{~dB}$. This is in good agreement with the apparent damping being reduced by a factor of 3.5 by a decreasing $\alpha$ from 1 to 0.25 , see Fig. 13(b). Further, it is seen that the strong spring-like behavior of the fuzzy at low frequencies dominates the response of the structure up to about $\Omega=140$. As the fuzzy is spring-like in the whole frequency range, it only causes the resonance frequencies of the structure to shift upwards. Fig. 14(b) shows the response at a position at some distance from the drive point. The damping effect of the fuzzy both without and with spatial memory is seen to be very similar to what was discussed earlier for the response at the drive point location. This illustrates and confirms that the influence of the structural fuzzy is global, and not specifically associated with the drive point.

\section{SUMMARY AND DISCUSSION}

Soize's method of including spatial memory in structural fuzzy has been thoroughly examined and exemplified in the present paper. Additional illustrations and a derivation of the fuzzy boundary impedance have been given in order to explain the ideas governing the method. To simplify the fuzzy modeling, Soize replaces the non-local spatial oscillator with a local equivalent oscillator. In the present article this oscillator has been given a physical interpretation. Further, it has been suggested that the so-called equivalent coupling factor, which transforms the nonlocal boundary impedance into a local impedance, can be determined as a function of the ratio between spatial memory and the free wavelength in the master.

The fuzzy boundary impedance, which includes spatial memory, has been derived deterministically by using a simple smoothing approach. This method assumes that the fuzzy is described in terms of a predefined distribution of resonating mass per unit frequency. The developed method is straightforward and it has been demonstrated that a prediction of the overall vibrations of the master can be made in a simple way.

From numerical simulations of the response of a simply supported beam with structural fuzzy and different amounts of spatial memory, it has been found that the spatial memory 
significantly reduces the damping introduced in the master. Further, for the case studied it can be included that the memory in some cases completely eliminates the damping effect of the fuzzy.

Various assumptions have been made in this paper in order to illustrate more clearly the effects of spatial memory in the structural fuzzy. This includes the hypothesis of modeling spatial memory by use of an equivalent spatial oscillator. A validation of this hypothesis and a discussion of its limitations clearly remain to be made. Also, a simple way of determining the equivalent coupling factor is as yet absent in the open literature. However, both of these two topics will be dealt with in a companion paper, which soon will be submitted for publication. Finally, further investigations are still required concerning practical questions of how one can determine the distribution of resonating mass per unit frequency as well as the amount of spatial memory in real-life engineering structures.

${ }^{1}$ J. Ormondroyd and J. P. Den Hartog, "Theory of dynamic vibration absorber," Trans. ASME 50, APM-241 (1928).

${ }^{2}$ Noise and Vibration, edited by R. G. White and J. G. Walker (Ellis Horwood, Chichester, UK, 1982), Chap. 25.

${ }^{3}$ D. F. Mead, Passive Vibration Control, (Wiley, Chichester, UK, 1999), Chap. 8.
${ }^{4}$ C. Soize, "Probabilistic structural modeling in linear dynamic analysis of complex mechanical systems, Part I," Rech. Aerosp. (English edition), 5, 23-48 (1986).

${ }^{5}$ F. Chabas, A. Desanti, and C. Soize, "Probabilistic structural modelling in linear dynamic analysis of complex mechanical systems, part II," Rech. Aerosp. (English edition), 5, 49-67 (1986).

${ }^{6} \mathrm{C}$. Soize, "A model and numerical method in the medium frequency range for vibroacoustic predictions using the theory of structural fuzzy," J. Acoust. Soc. Am. 94, 849-865 (1993).

${ }^{7}$ C. Soize, "Vibration damping in low-frequency range due to structural complexity. A model based on the theory of fuzzy structures and model parameters estimation," Comput. Struct. 58, 901-915 (1995).

${ }^{8}$ A. D. Pierce, V. W. Sparrow, and D. A. Russell, "Fundamental structuralacoustic idealizations for structures with fuzzy internals," J. Vibr. Acoust. 117, 339-348 (1995)

${ }^{9}$ M. Strasberg and D. Feit, "Vibration damping of large structures induced by attached small resonant structures," J. Acoust. Soc. Am. 99, 335-344 (1996).

${ }^{10} \mathrm{C}$. Soize, "Estimation of fuzzy substructure model parameters using the mean power flow equation of the fuzzy structure," J. Vibr. Acoust. 120, 279-286 (1998).

${ }^{11} \mathrm{C}$. Soize, "Estimation of fuzzy structure parameters for continuous junctions," J. Acoust. Soc. Am. 107, 2011-2020 (2000)

${ }^{12}$ A. D. Pierce, "Resonant-frequency-distribution of internal mass inferred from mechanical impedance matrices, with application to fuzzy structure theory," J. Vibr. Acoust. 119, 324-333 (1997).

${ }^{13}$ R. Cook, D. S. Malkus, M. F. Plesha, and R. J. Witt, Concepts and Applications of Finite Element Analysis (Wiley, New York, 2002). 